\title{
O apego ao lugar: uma abordagem no semiárido nordestino
}

\section{Place attachment: an approach in the northeastern semiarid}

\author{
Tony César de Sousa Oliveira ${ }^{1}$, Vanessa Fernanda da Silva Sousa ${ }^{2}$, Ruth Raquel Soares \\ de Farias ${ }^{3}$, Raimundo Nonato Lopes ${ }^{4}$, Antonio Alberto Jorge Farias Castro ${ }^{5}$ \\ 1 Doutorando em Biologia Comparada, USP, Ribeirão Preto, São Paulo, Brasil. Email: tonycsoliveira@hotmail.com, \\ ORCID: 0000-0001-6209-3829 \\ 2 Mestra em Desenvolvimento e Meio Ambiente, UFPI, Teresina, Piauí, Brasil. Email: vanessa_fernandass@hotmail.com,, \\ ORCID: 0000-0002-8591-5896 \\ 3 Doutora em Biotecnologia, Universidade Federal do Piauí, Teresina-PI, Brasil. Email: ruthraquelsf@gmail.com, ORCID: \\ 0000-0002-0988-0900 \\ 4 Graduando em Zootecnia, Universidade Federal do Piauí, Teresina-PI, Brasil. Email: rnlopes2008@ hotmail.com, ORCID: \\ 0000-0003-1641-610X \\ 5 Doutor em Biologia Vegetal, Universidade Federal do Piauí, Teresina-PI, Brasil. Email: albertojorgecastro@ufpi.edu.br, \\ ORCID: 0000-0002-2349-3843
}

\section{Recebido em 24/04/2019. Publicado em Dezembro/2019}

Palavras-chave: Afeto. Barragem. Pertencer. Território.

\section{Keywords:}

Affection. Dam.

Belonging. Territory.
RESUMO: A interação entre o indivíduo e o local provoca o surgimento de vínculos afetivos e, se essa relação é quebrada, isso pode causar grandes impactos ao indivíduo. Assim, este estudo propõe analisar as relações entre homem e lugar em uma comunidade que será afetada pela construção de uma barragem no estado do Piauí. Para isso, foram realizadas entrevistas com moradores da comunidade. Os dados foram analisados de forma qualitativa e qualitativa, utilizando-se o software IBM® SPSS® Statistic Software versão 20, e o software de análise textual de corpus (IRaMuTeQ, 0.6 alpha 3). O estudo revelou que os moradores da comunidade têm um forte vínculo afetivo com o local. Observamos também que os entrevistados demonstram insegurança em relação ao futuro, o que nos faz pensar sobre o processo de deslocamento de populações, incluindo informações sobre satisfação e apego ao local e a percepção da qualidade de vida

ABSTRACT: The interaction between the individual and the place may cause affective bonds and if this relationship is broken this can cause major impacts to the individual. Thus, this study proposed to analyze the relations between man and place in a community that will be affected by the construction of a dam in the state of Piauí. For this, interviews were conducted with community residents. The data were analyzed as qualitatively and qualitatively using the IBM® SPSS® Statistic Software version 20, and the textual corpus analysis software (IRaMuTeQ, 0.6 alpha 3). The study revealed that community residents have a strong affective bond with the place. We also observed that respondents show insecurity about the future, which makes us think about the process of displacement of populations including information about satisfaction and attachment to the place and the perception of the quality of life. 


\section{INTRODUÇÃO}

O termo 'lugar', como definido por Tuan (1977), é o ponto central de significados, que evidencia os sentimentos e as relações humanas, em um certo espaço. Ele é comumente utilizado para designar situações que se traduzem em hierarquia social, posição espacial, propriedade e privacidade (RAYMOND, BROWN; WEBER, 2010). Portanto, o 'lugar' não pode ser compreendido apenas em uma visão espacial, ele deve ser visto e pensando como um fenómeno holístico, onde se envolve questões sociais, psicológicas e ambientais (TUAN, 2018).

Estudos apontam que o apego ao lugar é chave principal para uma boa qualidade de vida, pois um lugar bem estruturado gera segurança e um sentimento de proteção ao indivíduo, levando a uma apropriação social, ocasionando um verdadeiro sentimento de pertencimento ao local (WIRTH et al., 2016). Acredita-se que essa apropriação vem da necessidade que o homem tem em possuir o seu próprio território e criar desse modo referências estáveis que o ajudem a orientar-se e preservar a identidade diante de si e dos outros (ANTON; LAWRENCE, 2014; WHEELER, 2017). Portanto, o lugar é algo de importância significativa para o indivíduo, pois este incorpora a própria identidade no lugar (TUAN, 2018).

Tramita-se hoje no estado do Piauí um projeto da construção de uma barragem no Rio Poti, que se situará entre os municípios de Juazeiro do Piauí, Castelo e Piauí e Buriti dos Montes, na região do Cânion do Poti. Apesar da grande contribuição de barragens para a vida humana, através da oferta de serviços essenciais (CHEN et al., 2016), essas construções ocasionam serias modificações no ambiente onde são estabelecidas (FEARNSIDE, 2016), principalmente no que tange o 'lugar' dos indivíduos (KIRCHHERR; CHARLES, 2016), uma vez que é no 'lugar' onde o homem mantém as suas interações sociais e naturais (RAYMOND; BROWN; WEBER, 2010).

Perante isso intencionou-se por meio deste estudo analisar as relações entre os indivíduos e o lugar em uma comunidade rural localizada na área de construção da Barragem de Castelo, no estado do Piauí, bem como aspectos socioeconômicos da população entrevistada. Existem algumas dimensões apontadas como constituintes do apego ao lugar: a dimensão social, onde o ser desenvolve uma afinidade, desenvolvendo assim uma dependência emocional com o local; a dimensão comunitária, onde o indivíduo desenvolve vínculos junto aos outros indivíduos que dividem o mesmo lugar; a dimensão natural, onde 
Ensino, Saúde e Ambiente - V12 (3), pp. 181-189, Dez. 2019

ocorre um apego ao ambiente, e o indivíduo desenvolve uma afinidade com o meio ambiente e aos fenômenos naturais (RAYMOND; BROWN; WEBER, 2010), nesse trabalho utilizaremos da dimensão social.

\section{METODOLOGIA}

A pesquisa foi realizada na comunidade rural Lagoa de Barro, município de Castelo do Piauí, distante $175 \mathrm{~km}$ da capital do estado do Piaú, Teresina, com localização situada nas coordenadas $05^{\circ} 10^{\prime} 20^{\prime}$ 'S, $41^{\circ} 42^{\prime} 12^{\prime}$ 'W e altitude média de 200 m.n.m. O município limita-se com os municípios piauienses de Pedro II ao norte, São Miguel do Tapuio ao sul, Juazeiro do Piauí, Alto Longá e São João da Serra Oeste e a leste com a área de litígio entre Piauí e Ceará (CEPRO, 2011). A seleção da amostra foi feita de forma probabilística abrangendo $100 \%$ das residências pertencentes à comunidade, onde para cada domicílio foram entrevistados dois adultos residentes (identificado com $\mathrm{P} n$, onde $\mathrm{P}$ significa participante e $\mathrm{n}$, um numeral identificador deste). Um total de nove casas foram visitadas, compreendendo uma amostra de 18 entrevistados.

O presente estudo foi aprovado pelo Comitê de Ética e Pesquisa (CEP) da Universidade Federal do Piauí (CAAE 66578317.4.0000.5214) e antes de sua aplicação, foram esclarecidos os objetivos da pesquisa e solicitado aos participantes que assinassem ou registrassem sua impressão digital junto ao Termo de Consentimento Livre e Esclarecido, segundo as exigências éticas do Conselho Nacional de Saúde por meio do Comitê de Ética em Pesquisa (Resolução 466/12). Para avaliar a relação com o lugar foram realizadas entrevistas com questões objetivas e subjetivas (BERNARD, 2006), com perguntas direcionadas a dimensões sociais, bem como sobre o perfil socioeconômico.

Os dados foram analisados de maneira quali-quantitativa. No tratamento dos dados se utilizou o Software IBM ${ }^{\circledR}$ SPSS $®$ Statistic versão 20. Foi ainda realizada a análise no Software IRaMuTeQ (Interface de R pour les Analyses Multidimensionnelles de Textes et de Questionnaires) 0.6 alpha 3, que permite fazer análises estatísticas sobre corpus textuais e sobre as transcrições das falas de uma entrevista.

\section{RESULTADOS E DISCUSSÕES}

Entre os entrevistados onze eram do gênero feminino e sete do gênero masculino e possuíam média de idade de 42 anos, sendo a predominância pessoas na faixa etária entre 30 a 70 anos. A maioria dos entrevistados possuía ensino fundamental incompleto (incluindo analfabetos e semianalfabetos) $(66,7 \%)$ (Tabela 1). Esse padrão encontrado é comum para localidades rurais, principalmente por essas comunidades serem formadas por pessoas de 
idades mais avançadas (PERES, 2011), como encontramos nesse estudo de caso. Essa população rural de idade mais avançada geralmente não teve acesso ao ensino básico devido a diversos motivos, principalmente históricos, tais como a falta de oportunidade, distanciamento de centros educacionais, entre outros (FERRARO, 2012). Isso pode ser observado em algumas falas dos entrevistados: "naquela época não tinha estudo, aprendemos foi trabalhando" p. 2, "nunca tive oportunidade de ler nem escrever, mas sou feliz assim" p. 14.

Sobre o estado civil, 14 dos entrevistados se declararam casados, dois viúvos e dois solteiros (Tabela 1). A renda média por pessoa é inferior a um salário-mínimo, tendo como principal fonte de renda os programas sociais (políticas públicas de transferência de renda) do governo federal e a atividade agropecuária. Um perfil bastante comum na região do nordeste brasileiro (VERAS, 2017), cujas famílias vivem de uma agropecuária de subsistência sem uma maior exploração de recursos naturais, ocasionada por diversos fatores, tais como o cultivo rudimentar e falta de tecnologia associados a fatores edáficos e climáticos (PERES, 2011; SUZART; FERREIRA, 2018).

Tabela 01- Avaliação dos dados socioeconômico dos residentes da comunidade Lagoa de Barro, Castelo do Piauí.

\begin{tabular}{lccc}
\hline VARIÁVEIS & $\begin{array}{c}\text { Número de } \\
\text { respostas }\end{array}$ & $\%$ & $\begin{array}{c}\text { P de significância } \\
\text { estatística }\end{array}$ \\
\hline
\end{tabular}

\section{Estado Civil}

Casado

$14 \quad 77,8 \%$

Viúvo

Solteiro

Separado

União estável

$0 \quad 0,0 \%$

\section{Escolaridade}

Fundamental incompleto ou analfabetos $12 \quad 66,7 \%$

Ensino fundamental completo

$4 \quad 22,2 \%$

Ensino médio completo

$2 \quad 11,1 \%$

Ensino superior

$0 \quad 0,0 \%$

\section{Atividade Profissional}

Trabalhador Rural

4

$22,2 \% \quad 0,0001 * * *$ 
Ensino, Saúde e Ambiente - V12 (3), pp. 181-189, Dez. 2019

\begin{tabular}{lcc}
\hline Vaqueiro & 5 & $27,8 \%$ \\
Domestica do lar & 9 & $50,0 \%$ \\
\hline
\end{tabular}

Fonte: Autores

Em relação ao tempo de moradia, $61,1 \%$ dos entrevistados relataram viver na comunidade há mais de 20 anos, e todos atribuem uma importância ao local, tais como: "Local que se tira o sustento" P 4; "Onde eu fui criada e criei meus filhos" P 8. Isso mostra um apego afetivo e social dos entrevistados ao lugar. Pode-se deduzir que nesse ambiente ocorreu a confirmação social de identidade do indivíduo (WHEELER, 2017), uma vez que os entrevistados apontaram o local de vivência como base da edificação pessoal de sua família. Esses fenômenos fazem com que o 'lugar' passe a ser um ambiente de efetividade onde os personagens estabelecem laços sentimentais, bem como um sentimento de pertencimento (LEWICKA, 2010).

Observou-se que todos os entrevistados declararam algum vínculo afetivo com o local (Tabela 2). Esse sentimento de afetividade pode ser explicado pela dimensão social, uma vez que os entrevistados demonstram ter estabelecido laços para com esse ambiente gerado principalmente porque esse lugar proporciona a satisfação das necessidades pessoais do indivíduo (RAYMOND; BROWN; WEBER, 2010), como pode-se observar nas seguintes falas transcritas: “Aqui 'nois' planta tudo e tudo dá, não precisamos sair daqui esse é um lugar que me faz bem" P 6, "Desde criancinha que eu vivo por essas bandas, aqui é onde eu me sinto feliz" P 2, "Esse lugar me lembra muito meu pai, foi ele que me ensinou todo o quanto eu sei" P 16.

Tabela 02- Sensação que os moradores da comunidade Lagoa de Barro sentem em relação ao seu espaço de vida.

\begin{tabular}{lccc}
\hline VARIÁVEIS & $\begin{array}{c}\text { Número de } \\
\text { respostas }\end{array}$ & $\%$ & $\begin{array}{c}\text { P de significância } \\
\text { estatística }\end{array}$ \\
\hline Sentimento de felicidade total & 18 & 100,0 & \\
Autoestima elevada & 18 & 100,0 & $<0,001$ \\
Sentimentos de gratidão & 18 & 100,0 & \\
Estado de humor geral & 18 & 100,0 & \\
Melhora da saúde global & 15 & 83,3 \\
Sentimentos de não solidão & 17 & 94,4 & \\
Satisfação com a vida em gera & 18 & 100,0 & \\
\hline
\end{tabular}


Ensino, Saúde e Ambiente - V12 (3), pp. 181-189, Dez. 2019

Sentimento de depressão

0

0,0

Fonte: Autores

Quando questionados sobre a perda do local em que vivem, foram observados os seguintes relatos: "Seria difícil se acostumar, pois já estamos acostumados aqui, e daqui tiramos tudo que precisamos" P 18, "Nem me fale nisso, aqui é onde eu crio meus bichos, cuido de minhas 'prantas' e vivo minha vidinha" P 5, "Seria ruim, pois aqui tem minhas criações” P 9, "Quando eu penso nisso me dá uma tristeza, eu não sei se seria bom e nem ruim, só sei que gosto de minha casinha" $\mathrm{P} 15$.).

Pode-se observar que o lugar passou a ser algo de importância significativa para os entrevistados, cujos incorporaram a própria identidade do lugar. Se ocorrer uma ruptura de elos entre o indivíduo e o seu lugar de convívio, poderá ocasionar grandes impactos sociais e psicológicos (PEREIRA; FERNANDES, 2011; WHEELER, 2017; TUAN, 2018). Isso pode ser observado também na nuvem de palavras gerada no Software Iramutec (Figura 1), que mostra que os entrevistados associam esse ambiente principalmente com a família e a subsistência (alimento).

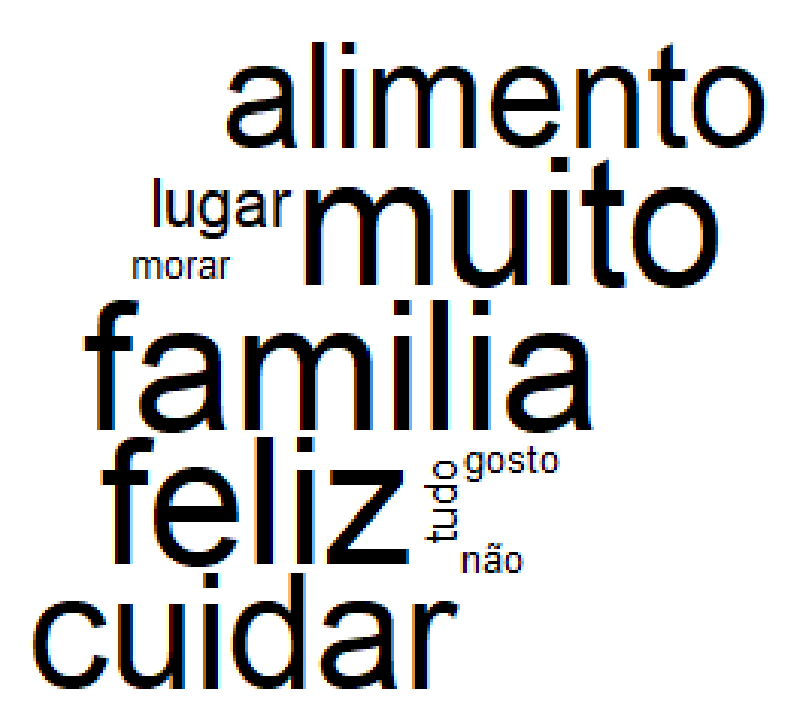

Figura 1- Palavras (com grafia adaptada para análise) mais utilizadas pelos moradores da comunidade Lagoa de Barro em relação à afetividade que eles possuem com o lugar.

Fonte: Autores

Quando solicitado aos entrevistados que descrevessem uma sensação que eles tinham em relação ao local que eles vivem (Tabela 3), todos expressaram sensações boas: “Aqui é um lugar que me traz paz" P 14, “Aqui eu me sinto bem, me faz muito bem” P 9, "Esse é o lugar 
Ensino, Saúde e Ambiente - V12 (3), pp. 181-189, Dez. 2019

que eu escolhi para viver o resto da minha vida" P 8, "Esse lugar é muito bom, foi onde eu criei meus meninos 'tudim"” P 4, “Esse lugar foi onde vivi a minha infância toda" P 8. As transcrições mostram um vínculo direto com um lugar, mostra também um ambiente como espaço transformador e afetivo, como habitat de boas lembranças, lugar como memória, isso demostra que o lugar foi incorporado na identidade do sujeito (TUAN, 2018).

Tabela 03- Nível de afetividade que os moradores da comunidade Lagoa de Barro sentem em relação ao ambiente.

\begin{tabular}{lccc}
\hline VARIÁVEIS & $\begin{array}{c}\text { Número de } \\
\text { respostas }\end{array}$ & $\%$ & $\begin{array}{c}\text { P de significância } \\
\text { estatística }\end{array}$ \\
\hline Lembranças boas da infância & 18 & 100 & \\
Lembrança de familiares & 18 & 100 & $<0,001$ \\
Lembranças boas & 17 & 94,4 & \\
Lembranças ruins & 1 & 5,6 & \\
Não gosta do local & 0 & 0 & \\
\hline
\end{tabular}

\section{Fonte: Autores}

Essa afetividade é influenciada principalmente pela memória, pensamento, percepção e inteligência, pois estes são bases na formação da personalidade humana e no estabelecimento de posse ao lugar em que estes estão inseridos (ANTON; LAWRENCE, 2014). Outro ponto a ser abordado é essa habilidade humana de sentir e expressar as suas sensações, sentimentos e emoções e por consequência a afetividade em relação ao lugar, faz parte desse processo de apropriação de um ambiente do qual lhe é retirado boas lembranças e recordações (VIDAL; VALERA; PERÓ, 2010).

\section{CONSIDERAÇÕES FINAIS}

Pôde-se observar que os entrevistados possuem um grande vínculo afetivo com esse ambiente. Esse apego ocasiona grande afetividade ao ambiente, seja material, cultural ou sentimental. Podemos destacar ainda que essa vinculação ao lugar é dada devido à representação deste como "repositório" de sentimentos, emoções e lembranças que dão sentido aos propósitos da vida dos indivíduos que utilizam esse determinado espaço. Quando construída a barragem, nos planos de realocação dessa comunidade, faz-se necessário então, na abordagem, a inclusão de informações sobre a satisfação e apego ao lugar e na percepção de qualidade de vida.

\section{REFERÊNCIAS}


Ensino, Saúde e Ambiente - V12 (3), pp. 181-189, Dez. 2019

ANTON, C. E.; LAWRENCE, C. Home is where the heart is: The effect of place of residence on place attachment and community participation. Journal of Environmental Psychology, v. 40, p. 451-461, 2014.

CHEN, J.; SHI, H.; SIVAKUMAR, B.; PEART, M. R. Population, water, food, energy and dams. Renewable and Sustainable Energy Reviews, v. 56, p. 18-28, 2016.

FEARNSIDE, P. M. Environmental and Social Impacts of Hydroelectric Dams in Brazilian Amazonia: Implications for the Aluminum Industry. World Development, v. 77, p. 48-65, 2016.

FERRARO, A. R. Alfabetização Rural no Brasil na Perspectiva das Relações Campo-Cidade e de Gênero. Educacao e Realidade, v. 37, p. 943-967, 2012.

KIRCHHERR, J.; CHARLES, K. J. The social impacts of dams: A new framework for scholarly analysis. Environmental Impact Assessment Review, v. 60, p. 99-114, 2016.

LEWICKA, M. What makes neighborhood different from home and city? Effects of place scale on place attachment. Journal of Environmental Psychology, v. 30, n. 1, p. 35-51, 2010 .

PEREIRA, C. J.; FERNANDES, D. Cultura e dimensões do viver em Yi-Fu Tuan: Algumas aproximações geográficas. RA'E GA - O Espaco Geografico em Analise, v. 22, n. 22, p. 53-73, 2011.

PERES, M. A. DE C. Velhice e analfabetismo, uma relação paradoxal: a exclusão educacional em contextos rurais da região Nordeste. Sociedade e Estado, v. 26, n. 3, p. 631662, 2011.

RAYMOND, C. M.; BROWN, G.; WEBER, D. The measurement of place attachment: Personal, community, and environmental connections. Journal of Environmental Psychology, v. 30, n. 4, p. 422-434, 2010.

SUZART, A. S.; FERREIRA, A. P. Avaliação do Programa Bolsa Família (PBF) na segurança alimentar e nutricional das famílias beneficiadas, Vitória da Conquista, BA. Interações (Campo Grande), v. 19, n. 3, p. 585, 2018.

TUAN, Y. Lugar: uma perspectiva experiencial 1. n. v, p. 4-15, 2018. 
VERAS, F. Brazil' s Bolsa Família : A Review Published by . Economic and Political Weekly v. 46, n. 21, p. 55-60, 2017.

VIDAL, T.; VALERA, S.; PERÓ, M. Apego al lugar, identidad de lugar and movilidad residencial en estudiantes de grado. Psyecology, v. 1, n. 3, p. 353-369, 2010.

WHEELER, R. Local history as productive nostalgia? Change, continuity and sense of place in rural England. Social \& Cultural Geography, v. 18, n. 4, p. 466-486, 2017.

WIRTH, T. VON; GRÊT-REGAMEY, A.; MOSER, C.; STAUFFACHER, M. Exploring the influence of perceived urban change on residents' place attachment. Journal of

Environmental Psychology, v. 46, p. 67-82, 2016.

\section{SOBRE OS AUTORES}

Este item só deve ser incluído na versão final, após a avaliação por pares.

AUTOR 1. Graduado em Ciências Biológicas e Mestre em Desenvolvimento e Meio Ambiente pela UFPI, hoje é aluno de doutorado em Biologia Comparada-USP. Desenvolve pesquisas em ecologia vegetal, diversidade funcional, ensino de biologia e ensino inclusivo.

AUTOR 2. Graduada em Ciências Biológicas e Mestra em Desenvolvimento e Meio ambiente pela UFPI, vem atuando principalmente em pesquisas relacionadas à botânica, ecologia e ensino de ciências e biologia.

AUTOR 3. Graduado em Zootecnia e especialista em Ovinocaprinocultura pelo Instituto de Ensino Superior Múltiplo (IESM). Tem experiência na área de Botânica e Ecologia, com ênfase em inventário das fitocomunidades do Cerrado e ecótonos associados.

AUTOR 4. Graduada em Ciências biológicas pela UFPI, Mestra em Biologia Vegetal pela UFPE e Doutora pelo programa de biotecnologia (RENORBIO - UFPI), atua nas seguintes frentes: biodiversidade, sustentabilidade, ambientalismo e educação ambiental.

AUTOR 5. Graduado Ciências Biológicas pela UFC (1978), Mestre e Doutor em Biologia Vegetal pena Unicamp, atualmente é professor titular da Universidade Federal do Piauí, atuando principalmente nas áreas de Botânica, Ecologia e Recursos Florestais. 